\title{
Monitoring mental health of college students using smartphones
}

\author{
Laura E. Barnes \\ lb3dp@virginia.edu / University of Virginia. Virginia, USA
}

Receipt: 15-8-2018 / Acceptance: 3-9-2018

\begin{abstract}
A better understanding of an individual's smartphone use can help researchers to improve methods for early detection, evaluation, and intervention of anxiety disorders. For this reason, we present an exploratory study of behavioral markers extracted from smartphone data. We examine fine-grained behaviors before and after smartphone communication events across social anxiety levels. To discover behavioral markers, we model the smartphone as a linear dynamical system with the accelerometer data as output. In a two-week study of 52 college students, we find substantially different behavioral markers prior to and after outgoing phone calls when comparing individuals with high and low social anxiety.
\end{abstract}

KEYWORDS: social anxiety, mental health, smartphones, mobile sensing, behavioral dynamics

\section{Monitoreo de la salud mental de estudiantes universitarios que emplean teléfonos inteligentes}

RESUMEN. Una mejor comprensión del uso del teléfono inteligente de un individuo puede ayudar a los investigadores a mejorar los métodos para la detección temprana, evaluación e intervención de trastornos de ansiedad. Por este motivo, presentamos un estudio exploratorio de marcadores de comportamiento extraídos a partir de los datos de teléfonos inteligentes. Examinamos los comportamientos específicos antes y después de los eventos de comunicación con teléfonos inteligentes asociados a los niveles de ansiedad social. Para descubrir los marcadores de comportamiento, modelamos el teléfono inteligente como un sistema dinámico lineal con los datos del acelerómetro como salida. En un estudio de dos semanas de duración realizado con 52 estudiantes universitarios, encontramos marcadores de comportamiento sustancialmente diferentes antes y después de las llamadas telefónicas salientes al comparar a personas con ansiedad social alta y baja.

PALABRAS ClAVE: ansiedad social, salud mental, teléfonos inteligentes, sensores para móviles, dinámica del comportamiento 


\section{INTRODUCTION}

Social anxiety is characterized by intense fear and avoidance of socially evaluative situations (American Psychiatric Association, 2013). Socially anxious individuals tend to exhibit behaviors (e.g., trembling, sweating, and fidgeting) consistent with this subjective state when a social interaction is perceived as threatening (Stein \& Stein, 2008). The experience of high social anxiety levels often results in severe avoidance of social interactions. Therefore, it is important to find behavioral markers indicating individuals' social anxiety.

Traditionally, psychological research on factors linked to social anxiety has relied on laboratory- based methods that limit the ecological validity of the findings. In contrast, recent advances have made it possible to passively monitor how behavioral systems unfold in people's natural settings by leveraging sensors embedded in personal smartphones (Huang et al., 2016; Chen et al., 2013). This approach has key advantages over traditional self-report surveys, which are subject to reporting bias, since behaviors suggestive of anxiety can be inferred passively and in situ.

However, naturally arising variations among smartphone user behaviors, and the link between physical and psychological dimensions of human behavior are not yet well understood.

We demonstrate passively sensed micromotions captured via smartphone accelerometers before and after phone call and text message communication events. We hypothesize that social anxiety levels are related to subtle differences in user motion before and after these communication events. We refer to these motions as the user's behavioral dynamics, and we use a linear dynamical system (LDS) to extract behavioral features from smartphone accelerometer data shortly before and after communication events. For phone calls, we also distinguish behaviors for outgoing and incoming calls. Using the extracted features, we then compare the effect sizes across social anxiety levels and communication types.

\section{Methodology}

With approval from the University of Virginia (UVA) Institutional Review Board, we recruited 52 undergraduate participants from the Psychology Department's participant pool. Participants received course credits as compensation. Before the study began, each participant was assessed on the Social Interaction Anxiety Scale (SIAS) (Mattick \& Clarke, 1998). The SIAS measures the long-term characteristic reactions to social interactions based on 20 items that are rated from 0 to 4 (e.g., "I have difficulty talking with other people."). Total SIAS scores range from 0 to 80 , with higher scores indicating greater anxiety associated with social interactions. Our participants' SIAS scores had a mean of 35.02 and a standard deviation of 12.10. In total, we collected 1,642 phone calls and 28,381 text messages from all the participants during the study period. 
During the initial lab session, we installed a general-purpose mobile sensing app called Sensus (Xiong, Huang, Barnes, \& Gerber, 2016) on each participant's personal smartphone to passively collect accelerometer data at $1 \mathrm{~Hz}$ as well as phone call and text message logs, all of which were uploaded to Amazon Web Services (AWS) Simple Storage Service (S3).

In our study, communication events referred to phone calls and text messages. To explore the effects of social anxiety on behavior around communication events, we first preprocessed the communication events. For phone calls, we distinguished outgoing calls and incoming calls, in which outgoing calls were individual initiated and planned social interactions while incoming calls were unexpected social interactions. Text messages were grouped as one event if two consecutive texts were fewer than 10 minutes apart. We could not distinguish the direction of text messages because phone numbers were randomly hashed to protect privacy of participants.

We preprocessed the accelerometer data into pre-event and post-event data indicating 10 minutes before a communication event happens and 10 minutes after a communication event finishes, respectively. The data within the pre-event and post-event observation time periods was then used to analyze behavioral dynamics. Figure 1 gives an example of a 9-minute outgoing phone call of a subject. It shows the accelerometer data during the pre- and post-call observation periods, which we used to analyze the behavioral dynamics before and after communication events. Before conducting further analysis of behavioral dynamics, we first applied a sliding window process to segment the accelerometer data into smaller, fixed-size chunks.

The details of this feature extraction (Huang et al., 2017) are shown in the lower half of Figure 1. The motion data in pre- and post-event observation periods are segmented into fixed sized sliding windows. The bottom half shows the feature extraction procedure using linear dynamic model and distance matrices.
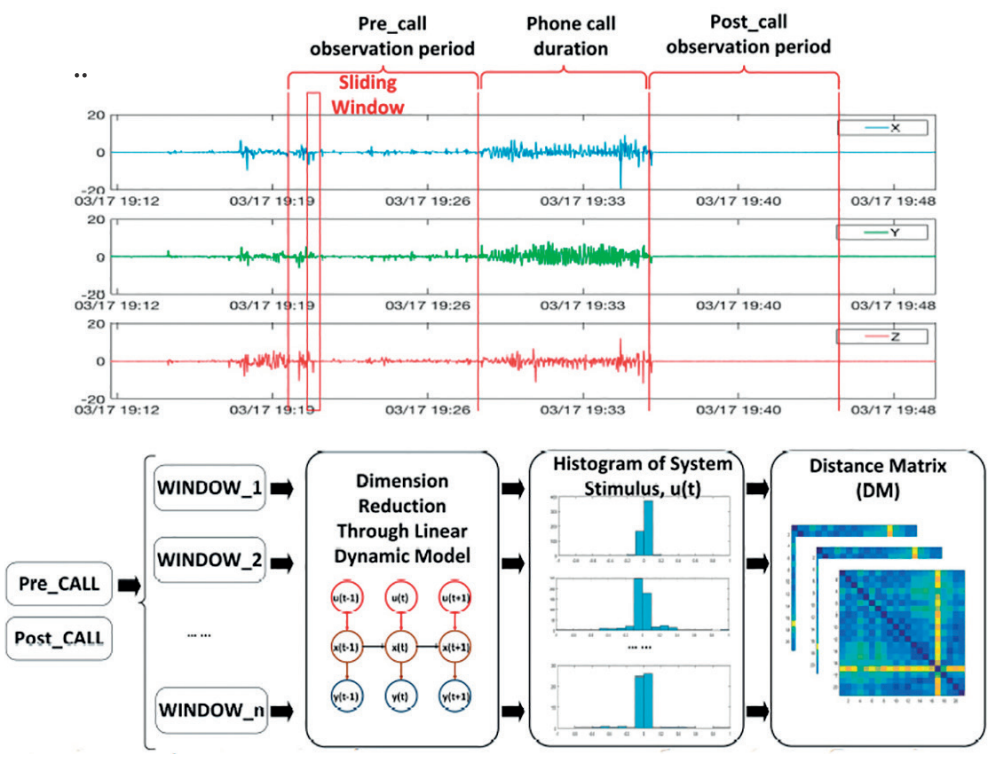

Figure 1. Diagram of the study design: an example of a 9-min outgoing call Source: Huang, et al., 2017 
After preprocessing and applying the linear dynamic model, we were able to reduce the high- dimensional raw temporal motion data to an array of low-dimensional features. These arrays were considered in a pairwise fashion to construct a distance matrix that uniquely characterized each subject's behavioral dynamics before and after a smartphone communication event.

\section{Results}

We used the features and metrics based on the approach described in Figure 1 for the behavioral dynamics analysis. We distinguished the behaviors before and after phone calls and text messages to explore the behaviors associated with social anxiety. Then we used effect-size analysis to discover the behavioral difference between low and high social anxiety groups. We also distinguished outgoing and incoming phone calls to further understand how individuals behave when they are going to initiate a social communication and when they have just ended a social communication event. In our study, we used Cohen's d to calculate the effect size. Generally, a $>0.5$ Cohen's $\mathrm{d}$ shows a medium effect size, while $>0.8$ shows a large effect size (Sawilowsky, 2009).

Table 1 summarizes the features and metrics used for the effect-size analysis. In the analysis, we grouped participants in a three-group setting using with a low-SIAS threshold and a high-SIAS threshold to split the participants into three groups: 1) low social anxiety risk, 2) medium social anxiety risk, and 3) high social anxiety risk. We analyzed the effect size between the low social anxiety risk group and the high social anxiety risk group. This setting provided a more nuanced grouping of the participants.

Table 1

Definition used for features and metrics

\begin{tabular}{|l|l|}
\hline Term & \\
\hline$\overline{F A C}_{1}$ & The average of the mean values of all distance matrices (DM(i)) belonging to a subject \\
\hline$\overline{F A C}{ }_{2}$ & $\begin{array}{l}\text { The average of the standard deviations of all distance matrices (DM(i)) belonging to a } \\
\text { subject }\end{array}$ \\
\hline MC_pre & The metric for the pre-event observation period of a phone call \\
\hline MC_post & The metric for the post-event observation period of a phone call \\
\hline MT_pre & The metric for the pre-event observation period of a text message group \\
\hline MT_post & The metric for the post-event observation period of a text message group \\
\hline
\end{tabular}

Source: Huang, et al., 2017 
In Table 2, two features are explored: 1) the average of all distance matrices' means belonging to one observation period (FAC1), and 2) the average of all distance matrices' standard deviations belonging to one observation period (FAC2). The results that have at least a medium effect size $(>=0.5)$ are shown in bold typeface.

Table 2

Effect-size analysis of the motion data before outgoing calls and after incoming calls between groups with (relatively) low versus high levels of social anxiety

\begin{tabular}{llcccc}
\hline & & \multicolumn{2}{c}{ Outgoing calls (MC_pre) } & \multicolumn{2}{c}{ Incoming calls (MC_post) } \\
\cline { 3 - 6 } SIAS low & SIAS high & $\overline{F A C}_{1}$ & $\overline{F A C}_{2}$ & $\overline{F A C}_{1}$ & $\overline{F A C}_{2}$ \\
\hline 27 & 44 & 0.2452 & 0.5234 & 0.3099 & 0.1748 \\
28 & 43 & 0.3007 & 0.5556 & 0.0835 & 0.2976 \\
30 & 39 & 0.2771 & 0.5490 & 0.2028 & 0.5103 \\
\hline
\end{tabular}

Source: Huang, et al., 2017

Our results suggest that behavioral metrics observed before outgoing phone calls have stronger associations with social anxiety scores than metrics observed after incoming phone calls. Our results also suggest that individuals in the low and high social anxiety groups behave distinctly before an active social interaction (when initiating a social interaction). These results may indicate that individuals with different social anxiety levels experience different levels of anxiety and associated behavioral variations (e.g., fidgeting and shaking) before calling others.

These findings may ultimately advance early detection, diagnosis, and the evaluation of treatment progress for social anxiety disorder.

\section{Conclusions}

Current methods to monitor social anxiety are usually based on retrospective self-report in the lab with little data to illuminate individuals' real-world behaviors. This paper presents a knowledge exploration study of the behavioral dynamics of smartphone use before and after phone calls and text messages. We created metrics to represent behavioral dynamics using accelerometer and communication history. We demonstrated that there is an observable difference in motions across social anxiety levels and that these behaviors further vary across different communication situations. This work opens up possibilities of passively monitoring behavioral markers of social anxiety. By passively sensing micro-level motion patterns, researchers and clinicians may better understand behavioral markers of social anxiety that can potentially be used to optimize treatment and intervention delivery. 


\section{REFERENCES}

American Psychiatric Association. (2013). Diagnostic and statistical manual of mental disorders (5th ed.).

Chen, Z., Lin, M., Chen, F., Lane, N. D., Cardone, G., Wang, R., ... \& Campbell, A. T. (2013). Unobtrusive sleep monitoring using smartphones. In Pervasive computing technologies for healthcare (pervasivehealth), 2013 7th international conference (pp. 145-152).

Huang, Y., Xiong, H., Leach, K., Zhang, Y., Chow, P., Fua, K., ... \& Barnes, L. E. (2016). Assessing social anxiety using gps trajectories and point-of-interest data. In Proceedings of the 2016 ACM international joint conference on pervasive and ubiquitous computing (pp. 898-903).

Huang, Y., Gong, J., Rucker, M., Chow, P., Fua, K., Gerber, M. S., ...\& Barnes, L. E. (2017). Discovery of behavioral markers of social anxiety from smartphone sensor data. In Proceedings of the 1st workshop on digital biomarkers (pp. 9-14). New York, NY, USA: ACM. DOI: 10.1145/3089341.3089343

Mattick, R. P. \& Clarke, J. (1998). Development and validation of measures of social phobia scrutiny fear and social interaction anxiety. Behaviour Research and Therapy, 36(4),455470. Retrieved from http://www.sciencedirect.com/science/article/pii/S000579679 7100316. DOI:10.1016/S0005-7967(97)10031-6

Sawilowsky, S. S. (2009). New effect size rules of thumb. Journal of Modern Applied Statistical Methods, 8(2), 597-599.

Stein, M. B. \& Stein, D. J. (2008). Social anxiety disorder. The Lancet, 371(9618), 1115-1125. DOI: $10.1016 /$ S0140-6736(08)60488-2

Xiong, H., Huang, Y., Barnes, L. E., \& Gerber, M. S. (2016). Sensus: A cross-platform, generalpurpose system for mobile crowdsensing in human-subject studies. In Proceedings of the 2016 ACM International Joint Conference on Pervasive and Ubiquitous Computing (pp. 415-426). New York, NY, USA: ACM. DOI: 10.1145/2971648.2971711 